\title{
ON A CLASS OF MINIMAL CONES IN $R^{n}$
}

\author{
BY PLINIO SIMOES ${ }^{1}$
}

Communicated by S. S. Chern, October 12, 1973

1. Introduction. In what follows let $S^{p}(\rho)=\left\{x \in R^{p+1}|| x \mid=\rho\right\}$ and $S_{p, q}=S^{p}\left((p /(p+q))^{1 / 2}\right) \times S^{q}\left((q /(p+q))^{1 / 2}\right) \subset S^{p+q+1}(1)$. Let $M$ be a codimension 1 , closed minimal submanifold of $S^{n+1}(1)$ and $C(M)=\{t x \mid 0<$ $t<1, x \in M\}$.

It is well known that $C(M)$ is a minimal submanifold of $R^{n+2}$. An important question is whether $C(M)$ minimizes area in $R^{n+2}$ with respect to its boundary $M$. With respect to this question the following results are known:

(a) When $n \leqq 5$, Simons [4] has given a negative answer.

(b) When $M=S_{p, p}, p \geqq 3$, Bombieri-De Giorgi-Giusti [1] have given an affirmative answer.

(c) When $M=S_{p, q}$ and either $p+q \geqq 7$ or $p=q=3$ Lawson [2], using a different approach from Bombieri-De Giorgi-Giusti, has given an affirmative answer.

(d) Lawson has also proved that when $n=6$ or $n=7$ the set of minimal cones $C(M)$, that minimize area in $R^{n+2}$ with respect to their boundary $M$, is finite up to diffeomorphisms.

In this note we answer the question when $M=S_{p, q}$ with $p+q=6$.

2. Results. Using techniques related to those of Bombieri-De GiorgiGiusti, we were able to prove in [3] the following two theorems:

THEOREM 1. If $p+q=n$ and either

(a) $n \geqq 7$ or

(b) $n=6$ with $|p-q| \leqq 4$,

then the cone $C\left(S_{p, q}\right)$ minimizes area in $R^{n+2}$ with respect to its boundary $S_{p, q}$.

THEOREM 2. $C\left(S_{1,5}\right)$ and $C\left(S_{5,1}\right)$ do not minimize area in $R^{8}$ with respect to their respective boundaries $S_{1,5}$ and $S_{5,1}$.

AMS (MOS) subject classifications (1970). Primary 28A75; Secondary 26A63, 35D10, 46Fxx, 53A10.

Key words and phrases. Area minimizing current, rectifiable current, oriented tangent cone, plateau problem.

${ }^{1}$ Partially supported by Conselho Nacional de Pesquisas (Brazil). 
Now let $V$ be a $C^{2}$ vector field in $R^{n+2}$, having compact support not containing $S^{n+1}(1)$ and let $\left\{\phi_{t}\right\}$ be its 1-parameter group of diffeomorphisms. We say that $C(M)$ is stable if for any such vector field there is $\varepsilon>0$ such that

$$
\text { Area of } \phi_{t}(C(M)) \geqq \text { Area of } C(M) \text { when }|t|<\varepsilon \text {. }
$$

By an argument similar to one in Simons [4] one may prove that $C\left(S_{1,5}\right)$ and $C\left(S_{5,1}\right)$ are stable.

So we have the following:

THEOREM 3. Although $C\left(S_{1,5}\right)$ and $C\left(S_{5,1}\right)$ are stable they do not minimize area in $R^{8}$ with respect to their respective boundaries $S_{1,5}$ and $S_{5,1}$.

\section{BIBLIOGRAPHY}

1. E. Bombieri, E. De Giorgi and E. Giusti, Minimal cones and the Bernstein problem, Invent. Math. 7 (1969), 243-268. MR 40 \#3445.

2. H. B. Lawson, Jr., The equivariant Plateau problem and interior regularity, Trans. Amer. Math. Soc. 173 (1972), 231-249.

3. P. Simoes, $A$ class of minimal cones in $R^{n}, n \geqq 8$, that minimize area, Ph.D. thesis, University of California, Berkeley, Calif., 1973.

4. J. Simons, Minimal varieties in riemannian manifolds, Ann. of Math. (2) 88 (1968), 62-105. MR 38 \#1617.

Instituto de Matematica, Universidade Federal do Ceara, Fortaleza, Ceara, BRAZIL 